\title{
Heat transfer increase with thin fins in three dimensional enclosures
}

\author{
R. L. Frederick \& S. Samper \\ Universidad de Chile, Departamento de Ingeniería Mecánica, \\ Santiago, Chile
}

\begin{abstract}
Heat transfer enhancement with highly conductive, thin vertical fins attached to the hot wall of a differentially heated cubical enclosure is numerically investigated. Two such fins attached to the hot wall were found to promote heat transfer enhancements of over $40 \%$ with respect to the unfinned case at $R a=10^{5}$ $-10^{7}$. This is one of the highest enhancements found to date in cavities of low aspect ratio. At a Rayleigh number of $10^{8}$, enhancements were lower, suggesting that this Rayleigh number marks the upper utilization limit of fins in enclosures to promote heat transfer. Results show that for further heat transfer optimization, sensitivity to variables as the fin thickness and length should be studied.
\end{abstract}

Keywords: natural convection, differentially heated cavities, fins.

\section{Introduction}

Although heat transfer in differentially heated cavities with fins on the active walls has been extensively studied, very few works have considered the three dimensional problem of a cubical cavity with vertical fins added to the hot wall. The extensive literature on the heat transfer effect of horizontal fins attached to one of the vertical active walls of rectangular enclosures [1-8] can be briefly summarized as follows: In low aspect ratio cavities, fins cause heat transfer reductions relative to the unfinned cavity at the same Rayleigh number if their thermal conductivity is low, while as thermal conductivity increases, fins cause moderate enhancements. Heat transfer tends to become insensitive to the solidto-fluid thermal conductivity ratio $\left(R_{k}=k_{s} / k_{f}\right)$ for $R_{k}>1000$ [4].

Simulations assuming fins of infinite thermal conductivity have been conducted in search of the highest possible heat transfer enhancements $[5,6]$. 
Even in this condition, with horizontal fins heat transfer is very insensitive to the fin length and to the height of the hot wall at which it is attached [5]. Very high heat transfer enhancements and reductions have been achieved recently, with thin fins of high and low thermal conductivity respectively [7, 8].

The heat transfer enhancement in 3D enclosures has been treated only recently. da Silva and Gosselin [9] studied a cubical enclosure with a parallelepiped fin on the hot wall. Frederick [10] recently provided numerical 3D results for a cavity with a thick vertical fin, with a thickness of 0.1 times the cavity side. Heat transfer enhancements of up to $30 \%$ for a fin with length equal to half the cavity side, and higher enhancements for longer fins, were obtained. Therefore, vertical fins were seen to be more effective that horizontal ones to promote heat transfer. The highest enhancements in [10] occurred at $R a \geq 10^{5}$ and $R_{k}>100$.

Heat transfer in a cubical enclosure with thin vertical fins attached to the hot wall is now studied. Thin fins add less weight and cause moderate perturbation to the flow field with respect to an undivided cavity. This gives the possibility of using double or multiple fins to increase heat transfer area with minimum flow blockage. However, thin fins have drawbacks: temperature may drop significantly along the fin when convection is intense. To characterize these effects we consider cavities with one or two vertical fins. To prevent excessive temperature drop along the fin, high $R_{k}$ values are used. The Rayleigh number range is $10^{5}-10^{8}$, making it possible to test the ability of the fins to produce heat transfer enhancement in situations in which convection is intense. The fin length and thickness are kept constant ( 0.5 and 0.01 times the cavity side respectively).

Heat transfer enhancements with respect to the unfinned cavity are reported. The flow patterns and temperature distributions with one and two fins are discussed to describe the interaction between two fins, and so anticipate if higher heat transfer increases can be obtained by placing more fins on the active walls. The results are physically interpreted in terms of isotherm diagrams.

\section{Problem formulation, numerical method and validation}

Consider an air- filled cubical cavity of side $L$ with two opposite vertical walls at imposed temperatures. Vertical conducting fins of height $L$ are attached to the hot wall $(X=0)$ which is at $T_{H}$, while the cold wall is at $T_{C}(X=1)$, where $T_{H}>T_{C}$. The other four walls are adiabatic. The Rayleigh number, based on $T_{H}-T_{C}$ and $L$, takes values of $10^{5}, 10^{6}, 10^{7}$, and $10^{8}$, and the dimensionless fin parameters (length $(l / L)$, thickness $(b / L)$ are kept constant at values of 0.5 and 0.01 respectively. The Prandtl number is also constant (0.71). The values of solid to fluid thermal conductivity ratio, $R_{k}$, are 100 and 1000 , representing materials of moderate to high thermal conductivity. The dimensionless equations of continuity, motion and energy in time dependent form with the Boussinesq assumption are:

$$
\frac{\partial U_{i}}{\partial X_{i}}=0
$$




$$
\begin{gathered}
\frac{\partial U_{i}}{\partial t}+U_{j} \frac{\partial U_{i}}{\partial X_{j}}=\frac{\partial p}{\partial X_{i}}+\operatorname{Pr} \frac{\partial^{2} U_{i}}{\partial X_{j} \partial X_{j}}+R a \operatorname{Pr} \Theta \delta_{i 2} \\
\frac{\partial \Theta}{\partial t}+U_{j} \frac{\partial \Theta}{\partial X_{j}}=\frac{\partial^{2} \Theta}{\partial X_{j} \partial X_{j}}
\end{gathered}
$$

Where $X_{1}=X, X_{2}=Y$ (vertical coordinate), $X_{3}=Z$. The thin vertical fins are attached to the hot wall at $Z=0.5$ if one fin is attached, and at $Z=1 / 3$ and $2 / 3$ for two fins attached. A dimensionless energy equation in the fin material is

$$
\frac{\partial \Theta}{\partial t}+\frac{\partial^{2} \Theta}{\partial X_{j} \partial X_{j}}=0
$$

At the cavity walls velocities $U, V$ and $W$ are zero. The dimensionless temperature $\Theta$ takes values of 0.5 and -0.5 at the hot and cold walls respectively. Adiabatic conditions $\partial \Theta / \partial Y=0$ and $\partial \Theta / \partial Z=0$ apply on the lateral and horizontal cavity walls. The fin faces are located at the following planes: $X=0$ (fin base), $X=l / L$ (fin tip), $Z=0.5 \pm b / 2 L$ (left and right faces in the case of one fin), $Z=1 / 3 \pm b / 2 L$ and $Z=2 / 3 \pm b / 2 L$ (two fins). The fin height equals the cavity height, that is, from $Y=0$ to $Y=1$. On all these faces zero velocities $(U=V=$ $W=0)$ are specified. The fin base $(X=0)$ is assumed to be at the hot wall temperature $(\Theta=0.5)$, while the thermal boundary conditions for its front face (Eq. (7)) and sides for one and two fins (Eqs. (5) and (6) respectively), express the continuity of the heat flux across the solid-fluid interfaces, as follows:

$$
\begin{gathered}
R_{k}\left(\frac{\partial \Theta}{\partial Z}\right)_{s}=\left(\frac{\partial \Theta}{\partial Z}\right)_{f} \quad Z=0.5 \pm b / 2 L \\
R_{k}\left(\frac{\partial \Theta}{\partial Z}\right)_{s}=\left(\frac{\partial \Theta}{\partial Z}\right)_{f} \quad Z=1 / 3 \pm b / 2 L \wedge Z=2 / 3 \pm b / 2 L \\
R_{k}\left(\frac{\partial \Theta}{\partial X}\right)_{s}=\left(\frac{\partial \Theta}{\partial X}\right)_{f} \quad X=l / L
\end{gathered}
$$

The numerical solution of the system is based on the SIMPLER method, with an in-house finite volume code that uses $100^{3}$ grid points and variable grid step. Although only steady state results are used, a false transient formulation of the governing equations is implemented to favour convergence and accuracy at high Rayleigh numbers. As high Rayleigh numbers were used, non uniform grids were adopted to concentrate grid points across the small fin thickness and on the cavity walls where the fields show high gradients. For validation, code results for Nusselt numbers in a cavity without fins were compared with simulations of 3D natural convection at high Rayleigh number $\left(10^{7}\right.$ and $\left.10^{8}[11,12]\right)$. The 
differences in the prediction of overall Nusselt number were always lower than $1 \%$. 16 fully three dimensional runs were performed.

\section{Results and discussion}

We discuss the overall, or 'cavity' heat transfer (Table 1). A general heat transfer increase relative to the unfinned case is observed, the increase being higher at higher thermal conductivity ratios, because on the fin surfaces a higher $R_{k}$ produces a more uniform temperature, which gets nearer to the hot wall temperature as $R_{k}$ grows. Nusselt number consistently increases with Rayleigh number, because of the higher intensity of convection which extracts heat from the hot wall and the fin faces.

Table 1: $\quad$ Cavity heat transfer: overall Nusselt numbers (16 runs).

\begin{tabular}{|c|c|c|c|c|c|}
\hline \multirow{2}{*}{ Ra } & \multicolumn{2}{|c|}{$R_{k}=100$} & \multicolumn{2}{c|}{$R_{k}=1000$} & \multirow{2}{*}{ No fin } \\
\cline { 2 - 5 } & 1 fin & 2 fins & 1 fin & 2 fins & \\
\hline $10^{5}$ & 4.678 & 5.134 & 5.145 & 6.140 & 4.337 \\
\hline $10^{6}$ & 9.450 & 10.274 & 10.391 & 12.011 & 8.617 \\
\hline $10^{7}$ & 17.887 & 19.476 & 19.567 & 22.534 & 16.164 \\
\hline $10^{8}$ & 31.941 & 33.681 & 35.734 & 38.667 & 30.242 \\
\hline
\end{tabular}

How effective is a thin fin compared to a thick fin? Although our previous results for a fin of thickness 0.1 [10] are for one fin only, and for Rayleigh numbers up to $10^{6}$, a direct comparison is possible for $R_{k}=1000$. The overall heat transfer with one thin fin is slightly lower $(10.8 \%$ and $8.85 \%$ decrease from the value for thick fin at $R a=10^{5}$ and $10^{6}$ respectively). A comparison of the maximum vertical velocities obtained for the case $R a=10^{6}$, is seen in Table 2 .

Table 2: $\quad$ Maximum vertical velocities with one thick and one thin fin.

\begin{tabular}{|l|c|c|c|}
\hline$R a$ & $V_{\max }$ (thick fin) & $V_{\max }($ thin fin) & $V_{\max }($ no fin) \\
\hline $10^{5}$ & 69.650 & 81.667 & 65.434 \\
\hline $10^{6}$ & 229.522 & 288.797 & 217.999 \\
\hline
\end{tabular}

A thick fin causes a slight flow increase compared to the finless case, as seen by the maximum vertical velocity. This is due to the increased area generating buoyancy forces. For the thin fin the increase is more pronounced, due to the lower hindrance on the flow by a thinner obstacle. Even this favourable flow condition produces a heat transfer reduction from the thin fin relative to the thick one. The reasons are two: apparently the temperature distribution along the thin fin is not as uniform as it is in the thick one, as Figure 2 shows, because of the increased conduction resistance along the thin fin. Therefore heat transfer from the fin sides is reduced. Another reason is that the heat transfer contribution of the fin front area (opposite the cold wall), which is considerable when using a 
thick fin, (not less than $10 \%$ of the total cavity heat transfer [10]), is not significant when the fin is very thin.

The above interpretation is confirmed when we consider the effect of the thermal conductivity of the fin. In Figure 1 overall heat transfer enhancements respect to the finless case are shown, for 1 and 2 fins and for both thermal conductivity ratios. An increase in $R_{k}$ from 100 to 1000 produces an average $9 \%$ increase in heat transfer for a single fin (except at $R a=10^{8}$, where the increase is lower $(5.44 \%)$. For double fins the increases are of about $16 \%$, and $8 \%$ at $R a=$ $10^{8}$.

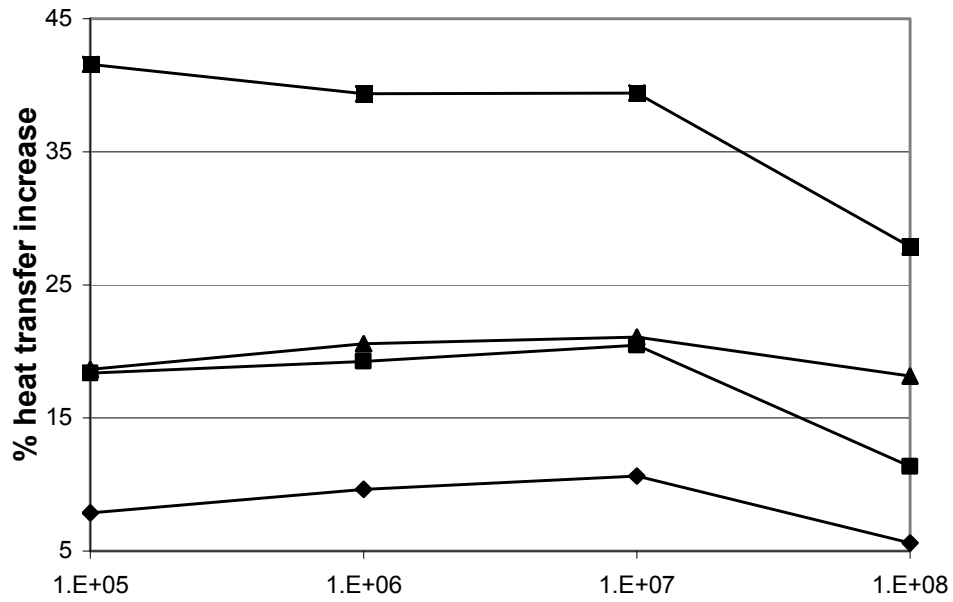

Figure 1: $\quad$ Percent increase in overall Nusselt number relative to the unfinned cavity. The curves, from top to bottom correspond to the following number of fins and $R_{k}$ values: 2-1000, 2-100, 1-1000, 1-100.

The preceding figure also illustrates how effective is the change from one to two fins. The maximum heat transfer enhancement obtained with two thin fins is $41 \%$. This is higher than the maximum enhancement obtained with a single thick fin in [10]. However, the heat transfer increase obtained in the two- fin situation compared to the one- fin situation with equal $R_{k}$ never exceeds $20 \%$ even with the highest thermal conductivity ratio. If the fins did not interact with each other the heat transfer increase could be doubled. There must, therefore, be some thermal interaction in the space between fins that tends to limit heat transfer.

Figure 2 shows isotherms in a plane containing one of the fins, for $\mathrm{Ra}=10^{8}$. Convection causes an appreciable temperature variation within the fin material, although most of the temperature range is in the right half of the figure (convective section) which is stratified, and shows a high temperature gradient at the cold wall. In the solid, the temperature is more uniform in the upper part, and the gradient is higher in the lower one, due probably to the incidence of cold fluid from the cold wall at $X=1$. Near the top the horizontal temperature gradient in the fin material is lower, indicating a more uniform temperature distribution. It is interesting to note that even if the fin material has a very high 


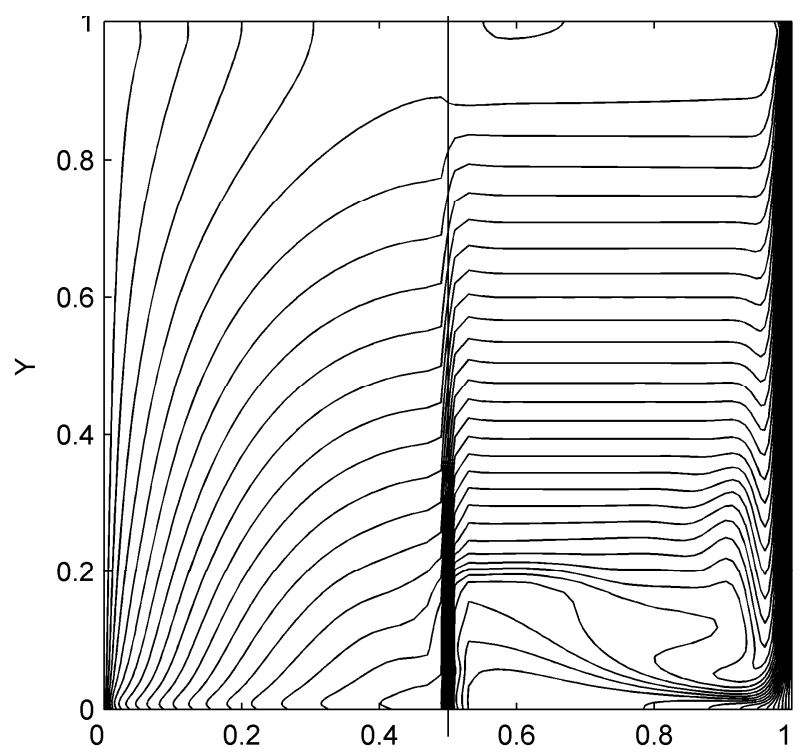

Figure 2: $\quad$ Isotherms in the plane of a single fin at $R_{k}=1000, R a=10^{8}$.

thermal conductivity, the convection at high Rayleigh number can be intense enough to produce a notorious temperature drop along $X$, as seen in Figure 2 .

Figure 3 shows temperature isograms at $Z=0.5$, which is the cavity midplane and also the mid-plane of the inter fin space, in a case with two fins. Although there is a thermal stratification, this is very different from the one in a cavity without fin. There is relative temperature uniformity in the upper half of the interfin space, as seen by the separation of isotherms. Uniformity comes from the fact that the fluid in the upper parts of the interfin space has already been heated by the internal fin faces. As seen in Figure 3, temperature gradients, and heat transfer from the upper part of the hot wall are greatly diminished. These facts account for the observed heat transfer increases with the number of fins.

Finally, the temperature isograms at the fin tips $X=0.5$ for one and two fins are shown (in Figures 4 and 5 , for $R a=10^{8}$ and $10^{7}$ respectively). Clearly, thermal stratification is seen, and the flows coming from the cold wall to the hot one are visualized by isotherms at the bottom of the enclosure. The cold wall flow is divided into two or three parallel streams for one and two fins respectively.

Significantly, the stream headed for the interfin space seems to be of lower intensity than the other two. As isotherms show, the heat transfer contribution is highest at the lower ends of the fins, where there is interaction with cold fluid coming from the cold wall. Figures 4 and 5 also show the thinning of the high temperature gradient zones around the fins as Ra grows. The fin faces are not shown in these figures for clarity.

Figure 1 reports the highest heat transfer enhancements yet obtained in cavities of low aspect ratio. Assuming that more than two fins will not produce 


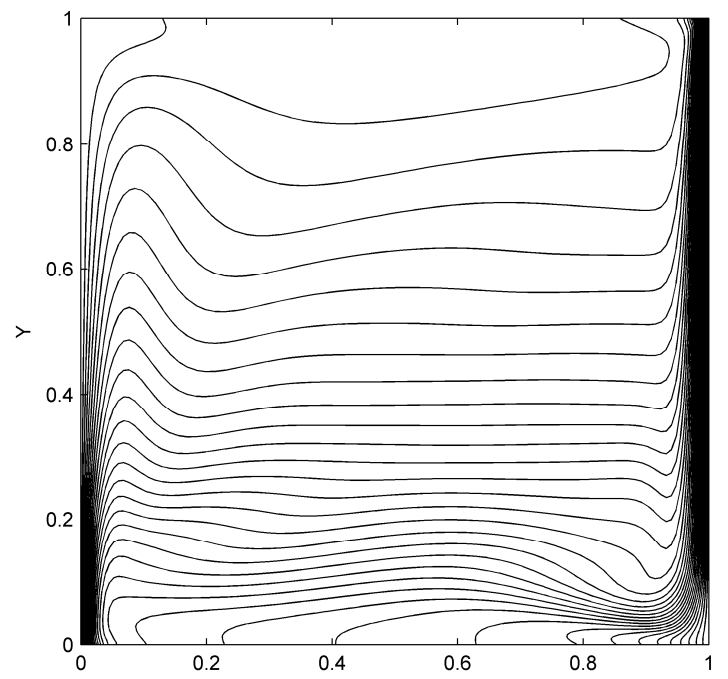

Figure 3: Isotherms at the inter-fin space for two fins, $R_{k}=1000, R a=10^{7}$.

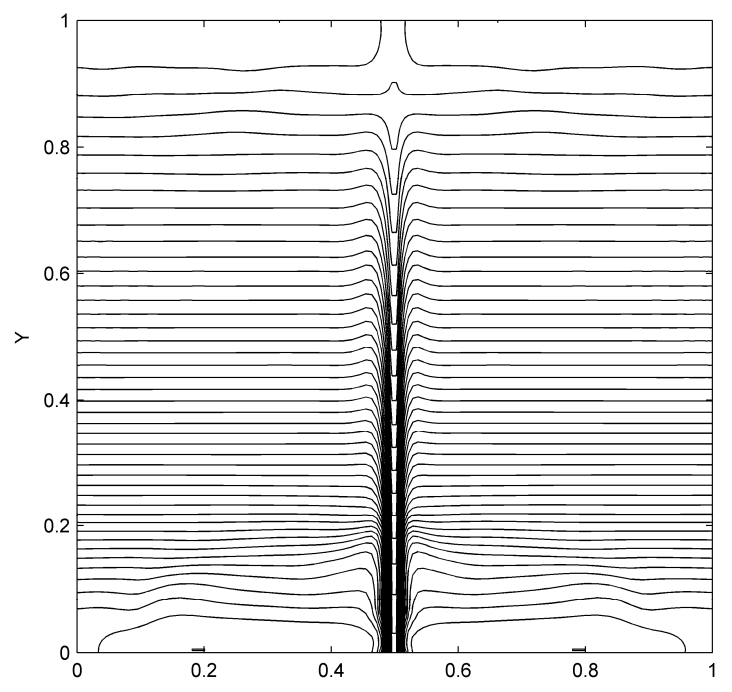

Figure 4: $\quad$ Isotherms at the fin tip for one fin, $R_{k}=1000, R a=10^{8}$.

equivalent heat transfer enhancements because of fin interaction, and also because the heat transfer tends to become asymptotic with $R_{k}$, it is interesting to provide an overall Nusselt number expression which is considered to be near the upper limit of heat transfer in a cubical enclosure:

$$
N u=0.29 R a^{0.267}
$$




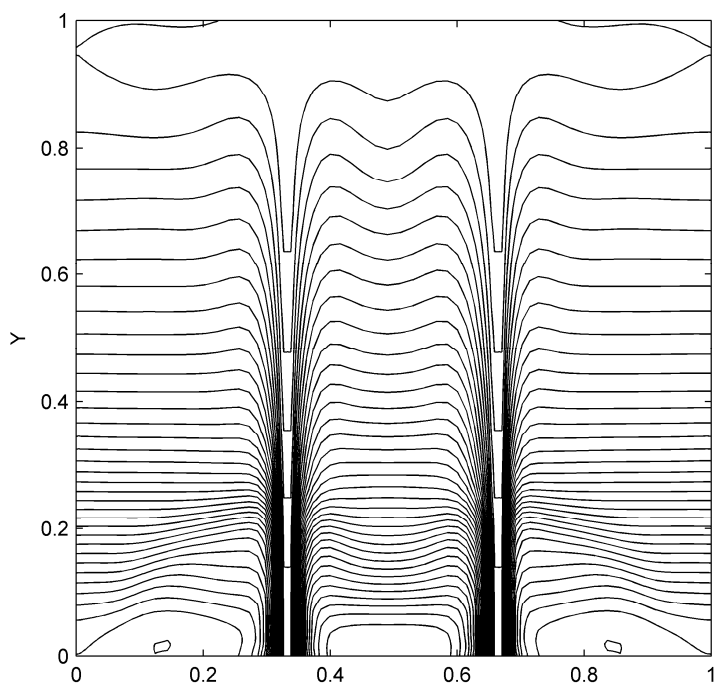

Figure 5: Isotherms at the fin tip for two fins, $R_{k}=1000, R a=10^{7}$.

The above expression is preliminary and corresponds to the case of two fins, with $R_{k}=1000$ and $10^{5} \leq R a \leq 10^{8}$. There are, however, several additional factors to consider if there is a need to maximize heat transfer in a cubical enclosure. An optimum fin thickness must be found, in close correspondence with a suitable number of fins. The optimum length of these elements must also be explored, as according to [10], heat transfer increases are expected beyond $l / L=0.5$

\section{Conclusions}

Heat transfer is always enhanced with high $R_{k}$ fins. Although a single thin fin does not promote heat transfer as much as a thick one, enhancements of over 40 percent with respect to the unfinned case are found with two fins at $R a=10^{5}$ $10^{7}$, which seem to be the highest found to date in cavities of low aspect ratio. While in the preceding range of $R a$, the temperature in the fin is nearly uniform, at a Rayleigh number of $10^{8}$, convection becomes so intense that even with $R_{k}=$ 1000 , there is an appreciable temperature drop along the fin, which limits the heat transfer increase to $28 \%$. This Rayleigh number therefore seems to mark the limit of utilization of fins in gas-filled enclosures to promote heat transfer. Temperature distributions denote thermal interaction between the two fins, so the number of fins to be used is limited by this interaction, which becomes detrimental to heat transfer. Therefore it is improbable that higher heat transfer increases can be obtained by placing more than two fins on the active walls. The most interesting variables for optimization of heat transfer with vertical fins seem to be the fin thickness and length. 


\section{References}

[1] Frederick, R.L., Natural convection in an inclined square enclosure with a partition attached to its cold wall. Int. J. Heat Mass Transfer, 32, pp. 87-94, 1989.

[2] Nag, A., Sarkar, A., Sastri, V.M.K., Natural convection in a differentially heated square enclosure with a horizontal partition plate on the hot wall. Computer Meth. in Appl. Mech. and Engng., 110, pp. 143-156, 1993.

[3] Frederick, R.L., Valencia, A., Heat Transfer in a square cavity with a conducting partition on its hot wall. Int. Commun. in Heat and Mass Transfer, 16, pp. 347-354, 1989.

[4] Nag, A., Sarkar, A., Sastri, V.M.K., Effect of a thick horizontal partial partition attached to one of the active walls of a differentially heated square cavity. Numerical Heat Transfer, Part A, 25, pp. 611-625, 1994.

[5] Shi, X., Khodadadi, J.M., Laminar natural convection heat transfer in a differentially heated square cavity due to a thin fin on the hot wall. ASME Journal of Heat Transfer, 125, pp. 624-634, 2003.

[6] Lakhal, E.K., Hasnaoui, M., Bilgen, E., Vasseur, P., Natural convection in inclined rectangular enclosures with perfectly conducting fins attached on the heated wall. Heat and Mass Transfer, 32, pp. 365-373, 1997.

[7] Tasnim, S.H., Collins, M.R., Numerical analysis of heat transfer in a square cavity with a baffle on the hot wall. Int. Commun. in Heat and Mass Transfer, 31, pp. 639-650, 2004.

[8] Bilgen, E., Natural convection in cavities with a thin fin on the hot wall. Int. J. Heat Mass Transfer 48, pp. 3493-3505, 2005.

[9] da Silva, A.K., Gosselin, L., On the thermal performance of an internally finned 3D cubic enclosure in natural convection. Int. Journal of Thermal Sciences, 44, pp. 540-546, 2005.

[10] Frederick, R.L., Heat transfer enhancement in cubical enclosures with vertical fins. Applied Thermal Engineering, 27, pp. 1585-1592, 2007.

[11] Tric, E., Labrosse, G., Betrouni, M., A first incursion into the 3D structure of natural convection of air in a differentially heated cubic cavity, from accurate numerical solutions. Int. J. Heat Mass Transfer, 43, pp. 40434056, 2000.

[12] Janssen, J.A., Henkes, R.A.W.M., Instabilities in three dimensional, differentially heated cavities with adiabatic horizontal walls. Phys. Fluids, 8, pp. 62-66, 1996. 